\title{
On a short spectral sum involving inner products of a holomorphic cusp form and Maass forms
}

\author{
by \\ Eeva Suvitie (Turku)
}

1. Introduction. We confine ourselves to cusp forms for the full modular group $\Gamma=\mathrm{SL}_{2}(\mathbb{Z})$ operating through Möbius transformations on the upper half-plane $\mathbb{H}=\{z=x+i y \in \mathbb{C} \mid y>0\}$. The standard fundamental domain $\mathcal{F}$ for $\Gamma$ is the region

$$
\{z=x+i y \in \mathbb{H}|| z \mid>1,-1 / 2<x<1 / 2\}
$$

together with its boundary in the half-plane $\operatorname{Re} z \leq 0$. A holomorphic cusp form $F(z): \mathbb{H} \rightarrow \mathbb{C}$ of weight $k \in \mathbb{Z}$ with respect to $\Gamma$ can be represented by its Fourier series

$$
F(z)=\sum_{n=1}^{\infty} a(n) e(n z), \quad e(\alpha)=\exp (2 \pi i \alpha) .
$$

We may assume that $k$ is even and $k \geq 12$, otherwise $F(z)$ is trivial.

A non-holomorphic cusp form or an automorphic form $u(z)=u(x+i y)$ : $\mathbb{H} \rightarrow \mathbb{C}$ is a non-constant real-analytic $\Gamma$-invariant function in the upper half-plane with the following properties:

(i) $u(z)$ is square-integrable with respect to the hyperbolic measure $d \mu(z)=d x d y / y^{2}$ over a fundamental domain of $\Gamma$.

(ii) $u(z)$ is an eigenfunction of the non-euclidean Laplacian $\Delta=$ $-y^{2}\left(\partial^{2} / \partial x^{2}+\partial^{2} / \partial y^{2}\right)$. The corresponding eigenvalue can be written as $1 / 4+\kappa^{2}$ with $\kappa>0$.

The Fourier series expansion for $u(z)$ is then of the form

$$
u(z)=y^{1 / 2} \sum_{n \neq 0} \rho(n) K_{i \kappa}(2 \pi|n| y) e(n x)
$$

2010 Mathematics Subject Classification: Primary 11F11, 11F12, 11F72.

Key words and phrases: holomorphic cusp forms, Maass wave forms, sums over short spectral intervals. 
with $K_{\nu}$ a Bessel function of imaginary argument. We may suppose that our cusp forms are eigenfunctions of the Hecke operators $T(n)$ for all positive integers $n$ and that $u(x+i y)$ is even or odd as a function of $x$. Thus $T(n) u=$ $t(n) u$ for certain real numbers $t(n)$, which are called Hecke eigenvalues, and $u(-\bar{z})= \pm u(z)$. Comparing Fourier coefficients on both sides, one may verify that $\rho(n)=\rho(1) t(n)$ and $\rho(-n)= \pm \rho(n)$ for all $n \geq 1$. See [Mo3] for proofs and for a general reference on non-holomorphic cusp forms.

Further, we define Petersson's inner product

$$
(f, g)=\int_{\mathcal{F}} f(z) \overline{g(z)} d \mu(z),
$$

which is well-defined for two square-integrable $\Gamma$-invariant functions. Now the Maass (wave) forms

$$
u_{j}(z)=y^{1 / 2} \sum_{n \neq 0} \rho_{j}(n) K_{i \kappa_{j}}(2 \pi|n| y) e(n x)
$$

constitute an orthonormal set of non-holomorphic cusp forms arranged so that the corresponding parameters $\kappa_{j}$ determined by the eigenvalues $1 / 4+$ $\kappa_{j}^{2}$ lie in an increasing order. We write $t_{j}(n)$ for the corresponding Hecke eigenvalues.

An interesting feature of holomorphic and non-holomorphic cusp forms is the analogy between their Fourier coefficients $a(n)$ and $\rho(n)$ and the classical divisor function $d(n)$. The analogy reveals itself for example in the Voronou type summation formulae, which are similar in each case (see [J1, Theorem 1.7] and [Me, Theorem 2]). One fascinating occurrence of the divisor function is in the additive divisor problem, in which one investigates the asymptotic behavior of the sum

$$
D(x ; m)=\sum_{n \leq x} d(n) d(n+m)
$$

as $x$ tends to infinity. Here $d(n)$ is the number of divisors of $n$, and $m$ is a given positive integer. Y. Motohashi's comprehensive paper [Mo1] gives an in-depth study of this problem along with a discussion of its history.

In his papers [J2] and [J3], M. Jutila considers generalizations of the additive divisor problem aiming at a unified approach to this sum along with its analogues

$$
A(x ; m)=\sum_{n \leq x} a(n) \overline{a(n+m)}
$$

over the Fourier coefficients of a holomorphic cusp form and

$$
T(x ; m)=\sum_{n \leq x} t(n) t(n+m)
$$


over the Hecke eigenvalues corresponding to Fourier coefficients of a nonholomorphic cusp form. Jutila studies these sums via the respective generating Dirichlet series aiming at analytic continuations of these series beyond the region of absolute convergence with estimates of at most polynomial order on vertical lines. The main complication turns out to be how to obtain the following estimates:

$$
\sum_{\kappa_{j} \leq K}\left|c_{j}\right|^{2} \exp \left(\pi \kappa_{j}\right) \ll K^{2 k+\varepsilon} \text { and } \sum_{\kappa_{j} \leq K}\left|\widetilde{c_{j}}\right|^{2} \exp \left(\pi \kappa_{j}\right) \ll K^{\varepsilon},
$$

where

$$
c_{j}=\left(u_{j}(z), y^{k}|F(z)|^{2}\right) \quad \text { and } \quad \widetilde{c}_{j}=\left(u_{j}(z),|u(z)|^{2}\right)
$$

are two Petersson's inner products involving Maass forms $u_{j}$ and the holomorphic and non-holomorphic cusp forms $F(z)$ and $u(z)$, respectively. (See [J2, Lemma 4].) The first of the above bounds was initially proved by A. Good in [G2], the argument being specific to holomorphic cusp forms, whereas Jutila developed a unified proof for both estimates in (1.1). Analogous estimates for inner products were established independently by P. Sarnak in [Sar], where he considers individual inner products of a more general type than those above, showing that $\left|\widetilde{c_{j}}\right|^{2} \exp \left(\pi \kappa_{j}\right)$ is of polynomial order in $\kappa_{j}$, although the order is weaker than what follows from the bound above.

In order to gain a deeper insight into the first of the $\kappa_{j}$-sums in (1.1) and into the order of a single inner product $c_{j}$, we study this sum over a short interval $K \leq \kappa_{j} \leq K+K^{1 / 3}$ and achieve the main result of this paper:

Main Theorem 1.1. For all $K \geq 1, \varepsilon>0$,

$$
\sum_{K \leq \kappa_{j} \leq K+K^{1 / 3}}\left|c_{j}\right|^{2} \exp \left(\pi \kappa_{j}\right) \ll K^{2 k-2 / 3+\varepsilon} .
$$

Here the implied constant depends on $k$ and $\varepsilon$.

This theorem along with its proof were included in our thesis [Su].

Note that the theorem is sharp up to the $\varepsilon$ in the exponent; see Good [G2, Remark 2 on p. 526] and A. Sankaranarayanan [San, Remark on p. 38].

Another of Jutila's papers [J4] serves as a motivation for choosing the cube root of $K$ as the length of the sum, as he investigates the sum

$$
\sum_{\left|\kappa_{j}-K\right| \leq K^{1 / 3}} \alpha_{j} H_{j}^{4}\left(\frac{1}{2}\right) \ll K^{4 / 3+\varepsilon} .
$$

Here $H_{j}(s)$ is the Hecke L-function attached to the $j$ th Maass form

$$
H_{j}(s)=\sum_{n=1}^{\infty} t_{j}(n) n^{-s},
$$


Re $s>1$, and $\alpha_{j}=\left|\rho_{j}\right|^{2} / \cosh \left(\pi \kappa_{j}\right)$ with $\rho_{j}=\rho_{j}(1)$ a Fourier coefficient of the corresponding Maass form. $H_{j}(s)$ can be analytically continued to an entire function in the complex plane $s \in \mathbb{C}$. The reason underlying this choice goes back to a paper by A. Ivić [Iv2], in which he proves that

$$
\sum_{\left|\kappa_{j}-K\right| \leq 1} \alpha_{j} H_{j}^{3}\left(\frac{1}{2}\right) \ll K^{1+\varepsilon},
$$

and as a corollary it follows that

$$
H_{j}\left(\frac{1}{2}\right) \ll \kappa_{j}^{1 / 3+\varepsilon} .
$$

We present a proof of Main Theorem 1.1 based on papers by Good G1] and Jutila and Motohashi [JM1, emphasizing those points where our argument deviates from these papers. In G1], Good proves that the inner product $c_{j}$ grows at most polynomially with respect to $\kappa_{j}$. He starts with the definition of the inner product, writes the holomorphic cusp form in question as a finite linear combination of Poincaré series and uses the Rankin-Selberg method to arrive at a single sum over Fourier coefficients $\rho_{j}(m)$ and $a(m)$. The result which Good thus obtains by direct estimations is not sharp enough for our purposes, so instead we continue along the lines of the approach used by Jutila and Motohashi. In [JM1] they treat an analogous sum with the divisor function $d(n)$ in place of the Fourier coefficient of a holomorphic cusp form, applying a series of various transformations and approximations to spectral and arithmetic objects. The key points in Jutila and Motohashi's proof for this sum are the use of a version of the Kloosterman-spectral sum formula of R. W. Bruggeman and N. V. Kuznetsov, the sum formula of Voronor, an explicit spectral decomposition of the shifted convolution sum and the spectral large sieve. In our case, some additional complications arise from the fact that Kloosterman sums appear instead of Ramanujan sums.

Although holomorphic cusp forms can be represented as finite linear combinations of Poincaré series, there is no analogous structure known for the non-holomorphic case. It is our future challenge to prove, in an analogous way to [Su, Chapter 3], a short interval estimate involving the inner products $\widetilde{c}_{j}$, that is,

$$
\sum_{K \leq \kappa_{j} \leq K+K^{1 / 3}}\left|\widetilde{c_{j}}\right|^{2} \exp \left(\pi \kappa_{j}\right) \ll K^{-2 / 3+\varepsilon},
$$

which requires a different approach. J. Bernstein and A. Reznikov have studied a more general case of the above in their papers [BR1] and [BR2]. They consider a product of two fixed Maass forms instead of the square of a fixed non-holomorphic cusp form to obtain a non-trivial bound $K^{-1 / 3+\varepsilon}$. 
In this paper, the following notation will be adopted: Generally we define

$$
\sigma_{\alpha}(n)=\sum_{d \mid n} d^{\alpha}
$$

as usual. Then $d(n)=\sigma_{0}(n)$ for all $n \in \mathbb{N}$. For any $\gamma=\left(\begin{array}{ll}a & b \\ c & d\end{array}\right) \in \Gamma$ we use the notation

$$
j(\gamma, z)=c z+d .
$$

As mentioned below 1.3), $\alpha_{j}=\left|\rho_{j}\right|^{2} / \cosh \left(\pi \kappa_{j}\right), \rho_{j}=\rho_{j}(1)$.

The Kloosterman sum is defined for the variables $m, n \in \mathbb{Z}, l \in \mathbb{Z}_{+}$by the formula

$$
S(m, n ; l)=\sum_{\substack{q=1 \\(q, l)=1}}^{l} e\left(\frac{m q+n \bar{q}}{l}\right), \quad q \bar{q} \equiv 1(\bmod l) .
$$

The non-holomorphic Eisenstein series is

$$
E(z, s)=\sum_{\gamma \in \mathcal{A}}(\operatorname{Im} \gamma(z))^{s}
$$

with $z \in \mathbb{H}$ and $\operatorname{Re} s>1$. Here $\mathcal{A}$ is a representative set of the right cosets $\Gamma_{\infty} \gamma$ in $\Gamma$, with $\Gamma_{\infty}=\{z \mapsto z+n \mid n \in \mathbb{Z}\}$ being the stabilizer of the cusp in $\Gamma$. E $(z, s)$ can be analytically continued to a meromorphic function in the complex plane $s \in \mathbb{C}$.

We define yet another inner product

$$
c(t)=\left(E(z, 1 / 2+i t), y^{k}|F(z)|^{2}\right)
$$

involving the non-holomorphic Eisenstein series $E(z, s)$ and the holomorphic cusp form $F(z)$.

We use the notation $m \sim M$ when $M \leq m<2 M$ and $m \asymp M$ when $A M \leq m \leq B M$ for some positive constants $A$ and $B$. Vinogradov's relation $f(z) \ll g(z)$ is another notation for $f(z)=\mathcal{O}(g(z))$. Since the implied constant in our Theorem 1.1 may depend on the weight $k$ and the arbitrarily small positive number $\varepsilon$, we shall often omit mentioning the dependence of the implied constants on those variables. In the context of complex integrals, the notation $\int_{(a)}$ means integration along the vertical line where the real part is $a$.

Further, we adopt Convention 2 from Jutila and Motohashi's paper [JM1]: Let $\mathcal{X}$ be a particular object that we need to bound and $\mathcal{Y}$ some expression that comes up in the course of the proof. Suppose we have an approximation $\mathcal{Y}=\mathcal{Y}_{0}+\mathcal{Y}_{1}+\mathcal{O}(\mathcal{Z})$, in which $\mathcal{Y}_{0}$ is dominant, $\mathcal{Y}_{1}$ oscillates in the same mode as $\mathcal{Y}_{0}$, and $\mathcal{Z}$ contributes negligibly to $\mathcal{X}$. Then clearly it suffices to treat only $\mathcal{Y}_{0}$ instead of $\mathcal{Y}$ and the notation $\mathcal{Y} \sim \mathcal{Y}_{0}$ indicates the use of a procedure in which the treatment of $\mathcal{Y}_{1}$ is a repetition of that of $\mathcal{Y}_{0}$. 
Notice that the notation $\sim$ is being used to mean different things in different places, but its meaning will be clear from the context. However, for clarity, we denote by $\approx$ the asymptotic expansions, usually also denoted by $\sim$.

We let $\varepsilon$ stand generally for a small positive number, not necessarily the same at each occurrence.

2. Some lemmas. We shall first gather some auxiliary results which will be used during the course of the proof of the Main Theorem.

A frequent role is played by the asymptotic representation of the Gamma function:

LEMma 2.1 (Stirling's formula). The following asymptotic expansion holds for the $\Gamma$-function:

$$
\Gamma(z) \approx \sqrt{2 \pi} \exp ((z-1 / 2) \log z-z)\left(1+\frac{1}{12 z}+\frac{1}{288 z^{2}}+\cdots\right),
$$

as $z \rightarrow \infty$ in the sector $|\arg z| \leq \pi-\delta$, with $\delta>0$ an arbitrary constant. In particular, in any fixed strip $b \leq \sigma \leq c$ we have

$$
|\Gamma(\sigma+i t)|=\sqrt{2 \pi}|t|^{\sigma-1 / 2} e^{-|t| \pi / 2}\left(1+\mathcal{O}\left(|t|^{-1}\right)\right)
$$

as $|t| \rightarrow \infty$.

For a proof, see Olver [O, p. 294].

Moreover, we have an asymptotic expansion involving Bernoulli numbers $B_{2 n}$ for the logarithm of the $\Gamma$-function:

$$
\begin{aligned}
\log \Gamma(z)= & (z-1 / 2) \log z-z+\frac{1}{2} \log (2 \pi) \\
& +\sum_{n=1}^{N} \frac{B_{2 n}}{2 n(2 n-1) z^{2 n-1}}+\mathcal{O}\left(\frac{1}{z^{2 N+1}}\right),
\end{aligned}
$$

when $|\arg z| \leq \pi-\delta$, with $N$ an arbitrary positive integer and $\delta>0$ an arbitrary constant. See [O, pp. 293-294].

For the Kloosterman sum we have the Weil bound

$$
S(m, n ; l) \ll d(l)(m, n, l)^{1 / 2} l^{1 / 2},
$$

see Estermann [Es, p. 86].

For the Kloosterman zeta-function we have the following spectral formula due to Kuznetsov (see [K, Lemma on p. 375]), originating from Selberg's paper [Se2]: 
Lemma 2.2. For $m, n \geq 1$ and $\operatorname{Re} s>3 / 4$,

$$
\begin{aligned}
& (2 \pi \sqrt{m n})^{2 s-1} \sum_{l=1}^{\infty} l^{-2 s} S(m, n ; l) \\
= & \frac{1}{2} \sin (\pi s) \sum_{j=1}^{\infty} \frac{\overline{\rho_{j}(m)} \rho_{j}(n)}{\cosh \left(\pi \kappa_{j}\right)} \Gamma\left(s-1 / 2+i \kappa_{j}\right) \Gamma\left(s-1 / 2-i \kappa_{j}\right)
\end{aligned}
$$$$
+\frac{1}{2 \pi} \sin (\pi s) \int_{-\infty}^{\infty} \frac{\sigma_{2 i r}(m) \sigma_{2 i r}(n)}{(m n)^{i r}|\zeta(1+2 i r)|^{2}} \Gamma(s-1 / 2+i r) \Gamma(s-1 / 2-i r) d r
$$$$
+\sum_{k=1}^{\infty}(2 k-1) q_{m, n}(k) \frac{\Gamma(k-1+s)}{\Gamma(k+1-s)}-\frac{1}{2 \pi} \delta_{m, n} \frac{\Gamma(s)}{\Gamma(1-s)}
$$

with

$$
q_{m, n}(k)=\sum_{l=1}^{\infty} \frac{1}{l} S(m, n ; l) J_{2 k-1}\left(\frac{4 \pi \sqrt{m n}}{l}\right),
$$

and $J_{\nu}$ the Bessel function of the first kind of order $\nu$. Here

$$
q_{m, n}(k) \ll(m n)^{\varepsilon}
$$

uniformly in $k$.

For a proof of equation (2.4), see Lemma 2.5 in [Mo3]. The estimate for $q_{m, n}(k)$ follows easily from Lemma 2.3 in Mo3 and Deligne's Theorem 8.2 of $[\mathrm{D}]$.

Moreover, we have the following estimate:

Lemma 2.3. Let $M, L \geq 1$ and let $n_{0}$ be a fixed positive integer. Let $g(l) \in C^{2}$ be a weight function with the property

$$
\operatorname{supp} g \subseteq[A L, B L]
$$

for some positive constants $A, B$, and let

$$
g^{(\nu)} \ll L^{-\nu} \quad \text { for } 0 \leq \nu \leq 2 .
$$

Let

$$
S=\sum_{m \asymp M}\left|\sum_{A L \leq l \leq B L} g(l) S\left(m, n_{0} ; l\right)\right|^{2} .
$$

Then $S \ll M L^{2+\varepsilon}$.

Proof. Clearly it suffices to prove that $S \ll M L^{2+\varepsilon}$ when $M \leq m \leq 2 M$. We use the notation $a_{m}=\sum_{A L \leq l \leq B L} \chi_{m} g(l) S\left(m, n_{0} ; l\right)$ with

$$
\chi_{m}= \begin{cases}1 & \text { if } M \leq m \leq 2 M \\ 0 & \text { otherwise }\end{cases}
$$


so that we can write $S$ in the form

$$
S=\sum_{M / 2 \leq m \leq 3 M / 2} g_{1}(m)\left|a_{m}\right|^{2}
$$

with $g_{1}$ a suitable $C^{2}$-function compactly supported on the interval $[M / 2$, $3 M / 2]$, and $g_{1}^{(\nu)} \ll_{\nu} M^{-\nu}$ for each $0 \leq \nu \leq 2$. Then

$$
S=\sum_{\substack{M / 2 \leq m \leq 3 M / 2 \\ N / 2 \leq n \leq 3 N / 2}} \chi_{m} \overline{a_{m}} \xi_{n} \sum_{A L \leq l \leq B L} g_{1}(m) g_{2}(n) g(l) S(m, n ; l)
$$

with $g_{2}$ a suitable $C^{2}$-function compactly supported on an appropriate interval $[N / 2,3 N / 2], N \geq 1$, and $g_{2}^{(\nu)} \ll 1$ for each $0 \leq \nu \leq 2, g_{2}\left(n_{0}\right)=1$ and

$$
\xi_{n}= \begin{cases}1, & n=n_{0} \\ 0, & n \neq n_{0}\end{cases}
$$

By Theorem 4 in [Iw1,

$$
S \ll L^{1+\varepsilon} M^{1 / 2} S^{1 / 2}
$$

and hence $S \ll M L^{2+\varepsilon}$.

Next we give some results related to cusp forms. First we have an important tool arising from spectral theory:

Lemma 2.4 (The spectral large sieve). For $K \geq 1,1 \leq \Delta \leq K, M \geq 1$ and any complex numbers $a_{m}$ we have

$$
\sum_{K \leq \kappa_{j} \leq K+\Delta} \alpha_{j}\left|\sum_{m \leq M} a_{m} t_{j}(m)\right|^{2} \ll(K \Delta+M)(K M)^{\varepsilon} \sum_{m \leq M}\left|a_{m}\right|^{2} .
$$

For a proof, see Theorem 1.1 in [J5] or Theorem 3.3 in [Mo3].

For the $\kappa_{j}$-sum with a single $m$ we have an exact leading term in

$$
\sum_{\kappa_{j} \leq K} \frac{\left|\rho_{j}(m)\right|^{2}}{\cosh \left(\pi \kappa_{j}\right)}=\frac{K^{2}}{\pi^{2}}+\mathcal{O}\left(K \log K+m^{\varepsilon} K+m^{1 / 2+\varepsilon}\right),
$$

as $\varepsilon>0, K \geq 2, m \geq 1$. For a proof, see Kuznetsov [K, Theorem 6]. As a consequence, for all $\varepsilon>0$ and $m \geq 1$ we have

$$
\rho_{j}(m) \ll \exp \left(\frac{\pi}{2} \kappa_{j}\right)\left(\kappa_{j}^{1 / 2}\left(\log \kappa_{j}\right)^{1 / 2}+m^{\varepsilon} \kappa_{j}^{1 / 2}+m^{1 / 4+\varepsilon}\right) .
$$

By choosing $m=1$ in 2.5 we have the following result: For all $K \geq 2$,

$$
\sum_{\kappa_{j} \leq K} \alpha_{j} \ll K^{2} .
$$


Moreover, we have the following estimate for a single $\alpha_{j}$ : For all $\varepsilon>0$,

$$
\alpha_{j} \ll \kappa_{j}^{\varepsilon}
$$

see Hoffstein and Lockhart [HL, Corollary 0.3].

Lastly, for the number of spectral parameters $\kappa_{j}$ in a certain range we have (see Hejhal [He, p. 511])

$$
N\left[0 \leq \kappa_{j} \leq K\right]=\frac{1}{12} K^{2}+\mathcal{O}(K \log K) .
$$

The next lemma is a continuous analogue of the spectral large sieve. (See also Iwaniec [Iw1, Theorem 3].)

Lemma 2.5. For $K$ real, $\Delta, M \geq 1$ and any complex numbers $a_{m}$ we have

$$
\begin{aligned}
\int_{K}^{K+\Delta}\left|\sum_{m \sim M} a_{m} \sigma_{2 i r}(m) m^{-i r}\right|^{2} d r & \ll\left(\Delta M^{1 / 2}+M\right) M^{\varepsilon} \sum_{m \sim M}\left|a_{m}\right|^{2} \\
& \ll\left(\Delta^{2}+M\right) M^{\varepsilon} \sum_{m \sim M}\left|a_{m}\right|^{2} .
\end{aligned}
$$

Note that the bounds are uniform in $K$.

Proof. We start from the inequality

$$
\int_{K}^{K+\Delta}\left|\sum_{m \sim M} a_{m} \sigma_{2 i r}(m) m^{-i r}\right|^{2} d r \leq \int_{-\infty}^{\infty}\left|\sum_{m \sim M} \sum_{d \mid m} a_{m} m^{-i r} d^{2 i r}\right|^{2} u(r) d r
$$

with $u$ a suitable smooth weight function compactly supported on an interval of length $\asymp \Delta$ and $u^{(\nu)} \ll_{\nu} \Delta^{-\nu}$ for each $\nu \geq 0$. By writing $m=d n$ and using a dyadic partition of the resulting $d$-sum we see that

$$
\begin{aligned}
\left|\sum_{m \sim M} \sum_{d \mid m} a_{m} m^{-i r} d^{2 i r}\right|^{2} & =\left|\sum_{1 \leq d<2 M} \sum_{n \sim M / d} a_{d n}\left(\frac{d}{n}\right)^{i r}\right|^{2} \\
& \ll \log ^{2} M\left|\sum_{d \sim D} \sum_{n \sim M / d} a_{d n}\left(\frac{d}{n}\right)^{i r}\right|^{2}
\end{aligned}
$$

for an appropriate $D$ in the range $1 / 2<D \leq M$. Hence the integral is

$$
\begin{aligned}
& \ll \log ^{2} M \int_{-\infty}^{\infty} \sum_{\substack{d_{1} n_{1} \sim M \\
d_{1} \sim D}} \sum_{\substack{d_{2} n_{2} \sim M \\
d_{2} \sim D}} a_{d_{1} n_{1}} \overline{a_{d_{2} n_{2}}}\left(\frac{d_{1}}{n_{1}}\right)^{i r}\left(\frac{d_{2}}{n_{2}}\right)^{-i r} u(r) d r \\
& \ll \log ^{2} M \sum_{d_{1}, n_{1}} \sum_{d_{2}, n_{2}}\left(\left|a_{d_{1} n_{1}}\right|^{2}+\left|a_{d_{2} n_{2}}\right|^{2}\right)\left|\int_{-\infty}^{\infty}\left(\frac{d_{1} n_{2}}{n_{1} d_{2}}\right)^{i r} u(r) d r\right| .
\end{aligned}
$$


We next study the last integral, using the abbreviation

$$
A=\frac{d_{1} n_{2}}{n_{1} d_{2}} .
$$

Now trivially

$$
\int_{-\infty}^{\infty} A^{i r} u(r) d r \ll \Delta
$$

and by integration by parts

$$
\int_{-\infty}^{\infty} A^{i r} u(r) d r=\frac{(-1)^{\nu}}{(i \log A)^{\nu}} \int_{-\infty}^{\infty} A^{i r} u^{(\nu)}(r) d r \ll_{\nu} \Delta M^{-\nu \varepsilon}
$$

if $|A-1| \gg M^{\varepsilon} / \Delta$. Then the sum involving terms for which this condition holds has the desired bound. Therefore, by symmetry, it is sufficient to consider the sum

$$
\log ^{2} M \sum_{\substack{d_{1}, n_{1} \\ d_{2}, n_{2}}}\left|a_{d_{1} n_{1}}\right|^{2} \Delta,
$$

where the condition of summation is

$$
\frac{d_{1} n_{2}}{n_{1} d_{2}}=1+\mathcal{O}\left(\frac{M^{\varepsilon}}{\Delta}\right)
$$

that is,

$$
\frac{d_{1}}{n_{1}}=\frac{d_{2}}{n_{2}}+\mathcal{O}\left(\frac{D M^{\varepsilon}}{N \Delta}\right)
$$

with $N=M / D$. Further, by symmetry, we may assume that $N \ll M^{1 / 2}$. Now if we fix $d_{1} / n_{1}$, then there are $\ll N$ possibilities for $n_{2}$, and, for each $n_{2}$, $\ll 1+D M^{\varepsilon} / \Delta$ possibilities for $d_{2}$. Finally we have the upper bound

$$
\begin{aligned}
& \ll \log ^{2} M \sum_{m \sim M} d(m)\left|a_{m}\right|^{2} \Delta N\left(1+\frac{D M^{\varepsilon}}{\Delta}\right) \ll(\Delta N+M) M^{\varepsilon} \sum_{m \sim M}\left|a_{m}\right|^{2} \\
& \ll\left(\Delta M^{1 / 2}+M\right) M^{\varepsilon} \sum_{m \sim M}\left|a_{m}\right|^{2} \ll\left(\Delta^{2}+M\right) M^{\varepsilon} \sum_{m \sim M}\left|a_{m}\right|^{2} .
\end{aligned}
$$

Well-known estimates for all Fourier coefficients of a holomorphic cusp form state that

$$
|a(n)|^{2} \ll n^{k-1+\varepsilon}, \quad \sum_{n \leq N}|a(n)|^{2}=A N^{k}+\mathcal{O}\left(N^{k-2 / 5}\right)
$$

with $A$ a positive constant. For a proof of the first estimate, see Deligne [D, Theorem 8.2], and for the second one, see Rankin [R, Theorem 1] or Selberg [Se1, p. 49].

Analogously to Maass forms with the spectral parameter $\kappa$ we have the following estimate due to Iwaniec [Iw2, Lemma 1]: For all $\varepsilon>0$ and $N \geq 1$ 
we have

$$
\sum_{n \leq N} t^{2}(n) \ll \kappa^{\varepsilon} N
$$

In [J2, Lemma 3], Jutila has shown that for all $K \geq 1$,

$$
\int_{-K}^{K}|c(u)|^{2} \exp (\pi|u|) d u \ll K^{2 k+\varepsilon}
$$

with $c(u)$ defined in $(1.6)$. The result is not the best known, but suffices for our purposes.

Next we have a formula for the spectral decomposition of the shifted convolution sum over the Fourier coefficients of a holomorphic cusp form, due to Motohashi, Mo2].

Lemma 2.6. Let $f$ be a positive integer and $W$ a smooth function of compact support on $(0, \infty)$. Then

$$
\begin{aligned}
\sum_{l=1}^{\infty} \overline{a(l)} a(l & +f) W\left(\frac{l}{f}\right) \\
= & i \pi(4 \pi)^{k-1} f^{-1 / 2+k}\left(\sum_{j=1}^{\infty} \overline{\rho_{j}} t_{j}(f) c_{j} \Phi_{k}\left(\kappa_{j} ; W\right)\right. \\
& \left.+\frac{1}{2 \sqrt{\pi}} \int_{-\infty}^{\infty} \frac{\sigma_{2 i r}(f) c(r)}{(\pi f)^{i r} \Gamma(1 / 2-i r) \zeta(1-2 i r)} \Phi_{k}(r ; W) d r\right)
\end{aligned}
$$

with $c_{j}$ as in (1.2) and $c(r)$ as in (1.6) the inner products attached to the holomorphic cusp form in question, and

$$
\begin{aligned}
& \Phi_{k}(r ; W)=\frac{1}{\sinh (\pi r)} \frac{\Gamma(1 / 2+i r)}{\Gamma(k-1 / 2-i r) \Gamma(1+2 i r)} \\
& \times \int_{0}^{\infty} y^{1 / 2-k-i r} W(y)(y(y+1))^{k-1} F(k-1 / 2+i r, 1 / 2+i r ; 1+2 i r ;-1 / y) d y \\
& +(r \mapsto-r) .
\end{aligned}
$$

Here $F(\cdot, \cdot ; \cdot ; \cdot)$ is the hypergeometric function and $(r \mapsto-r)$ stands for an expression similar to the preceding one, but with $r$ replaced by $-r$.

This follows easily from Jutila and Motohashi's result [JM2, Lemma 4]. We notice that in [JM2], the lemma has been formulated under the assumption that $F(z)$ is a Hecke eigenform. This is, however, not essential. Note that $\overline{a(l)} a(l+f)$ in the above lemma can be replaced by $a(l) \overline{a(l+f)}$. Further, if the support of $W$ tends to infinity, then

$$
F(k-1 / 2+i r, 1 / 2+i r ; 1+2 i r ;-1 / y) \sim\left(\frac{1+\sqrt{1+y^{-1}}}{2}\right)^{1-2 k-2 i r}
$$


by the quadratic transformation formula for the hypergeometric function from Lebedev [L, eq. (9.6.12)].

Lastly, the following identity is a refined version of the Kloostermanspectral sum formula of Bruggeman and Kuznetsov:

LEMma 2.7. Let $h(r)$ be an even and regular function in the strip $|\operatorname{Im} r|$ $<1 / 4+\varepsilon$, and there $|h(r)| \ll(1+|r|)^{-2-\varepsilon}$. Put

$$
\hat{h}(x)=\frac{2 i}{\pi} \int_{-\infty}^{\infty} \frac{r h(r)}{\cosh (\pi r)} J_{2 i r}(x) d r=\frac{2 i}{\pi} \int_{0}^{\infty} \frac{r h(r)}{\cosh (\pi r)}\left(J_{2 i r}(x)-J_{-2 i r}(x)\right) d r,
$$

with $J_{\nu}$ again the Bessel function of the first kind of order $\nu$. Then

$$
\begin{aligned}
& \sum_{j=1}^{\infty} \alpha_{j} t_{j}(m) t_{j}(n) h\left(\kappa_{j}\right)=-\frac{1}{\pi} \int_{-\infty}^{\infty} \frac{\sigma_{2 i r}(m) \sigma_{2 i r}(n)}{(m n)^{i r}|\zeta(1+2 i r)|^{2}} h(r) d r \\
&+\frac{\delta_{m, n}}{\pi^{2}} \int_{-\infty}^{\infty} r \tanh (\pi r) h(r) d r+\sum_{l=1}^{\infty} \frac{1}{l} S(m, n ; l) \hat{h}\left(\frac{4 \pi \sqrt{m n}}{l}\right),
\end{aligned}
$$

where $\delta_{m, n}$ is the Kronecker delta.

For a proof, see [Mo3, §2.6].

3. Proof of the Main Theorem. In [G1, Lemma 2], Good first represents $c_{j}$ as a finite linear combination of terms of the form

$$
\gamma_{n, n^{\prime}}(j)=\int_{\mathcal{F}} y^{k-2} P_{n}(z, k) \overline{P_{n^{\prime}}(z, k)} u_{j}(z) d x d y
$$

with $n, n^{\prime} \in \mathbb{Z}_{+}$and

$$
P_{n}(z, k)=\sum_{\gamma \in \mathcal{A}} j(\gamma, z)^{-k} e(n \gamma(z)), \quad z \in \mathbb{H}, n, k \in \mathbb{N}
$$

the Poincaré series, $j(\gamma, z)$ as in (1.4) and $\mathcal{A}$ as in $(1.5)$. He then ends up with equation (4.9) in his paper which states that

$$
\begin{aligned}
\gamma_{n, n^{\prime}}(j)= & \left(\frac{\pi}{2}\right)^{1 / 2} \Gamma\left(k-s_{j}\right) \Gamma\left(k-\overline{s_{j}}\right) \sum_{\substack{m>-n \\
m \neq 0}} \rho_{j}(m) \overline{a(m+n)} \\
& \times(2 \pi|m|)^{-k+1 / 2}\left(\frac{4 n^{2}+4 n m}{m^{2}}\right)^{(1-k) / 2} \mathcal{P}_{s_{j}-1}^{1-k}\left(\frac{2 n+m}{|m|}\right)
\end{aligned}
$$

with $s_{j}=1 / 2+i \kappa_{j}, a(l)$ the $l$ th Fourier coefficient of the Poincaré series $P_{n^{\prime}}(z, k)$ and $\mathcal{P}_{\nu}^{\mu}$ the Legendre function of the first kind.

We estimate the $\Gamma$-functions in (3.1) by Stirling's formula and notice that for the proof of our Main Theorem 1.1 it suffices to prove that for all 
fixed $n \in \mathbb{Z}_{+}$,

$$
\begin{aligned}
\left.\sum_{K \leq \kappa_{j} \leq K+K^{1 / 3}} \alpha_{j}\left|\sum_{\substack{m>-n \\
m \neq 0}} t_{j}(m) \overline{a(m+n)}\right| m\right|^{-1 / 2}(\sqrt{n(n+m)})^{1-k} \\
\quad \times\left.\mathcal{P}_{s_{j}-1}^{1-k}\left(\frac{2 n+m}{|m|}\right)\right|^{2} \ll K^{-2 k+3+1 / 3+\varepsilon} .
\end{aligned}
$$

Good carries on to represent the Legendre function in terms of the hypergeometric function and derives asymptotic expansions (4.18) and (4.19) which yield

$$
\mathcal{P}_{s_{j}-1}^{1-k}\left(\frac{2 n+m}{|m|}\right) \sim \frac{1}{(k-1) !}\left(\frac{n}{n+m}\right)^{(k-1) / 2}
$$

if $m \gg K^{2+\varepsilon}$, and

$$
\begin{array}{r}
\mathcal{P}_{s_{j}-1}^{1-k}\left(\frac{2 n+m}{|m|}\right) \sim \frac{\sqrt{|m|}}{2 \sqrt{\pi}(n(n+m))^{1 / 4}}\left\{\frac{\Gamma\left(-i \kappa_{j}\right)}{\Gamma\left(k-1 / 2-i \kappa_{j}\right)}\right. \\
\quad \times \exp \left(-i \kappa_{j} \log \left(\frac{m+2 n+2 \sqrt{n(n+m)}}{|m|}\right)\right) \\
\left.+\frac{\Gamma\left(i \kappa_{j}\right)}{\Gamma\left(k-1 / 2+i \kappa_{j}\right)} \exp \left(i \kappa_{j} \log \left(\frac{m+2 n+2 \sqrt{n(n+m)}}{|m|}\right)\right)\right\}
\end{array}
$$

if $-n+1 \leq m \ll K^{2-\varepsilon}, m \neq 0$.

If we are in the "transitional area" $K^{2-\varepsilon} \ll m \ll K^{2+\varepsilon}$, then these asymptotic expansions do not apply, and we use the following formula:

$$
\mathcal{P}_{s-1}^{1-k}(x)=\frac{\pi^{-1 / 2} 2^{1-k}\left(x^{2}-1\right)^{(k-1) / 2}}{\Gamma(k-1 / 2)} \int_{0}^{\pi}\left(x+\left(x^{2}-1\right)^{1 / 2} \cos t\right)^{s-k}(\sin t)^{2 k-2} d t,
$$

which holds for all $k>1 / 2$ and $x>1$ (see [EMOT, eq. (6) on p. 155]). Therefore we have

$$
\begin{aligned}
\mathcal{P}_{s_{j}-1}^{1-k}\left(\frac{2 n+m}{|m|}\right) & =\pi^{-1 / 2}(\Gamma(k-1 / 2))^{-1}\left(\frac{n(n+m)}{m^{2}}\right)^{(k-1) / 2} \\
& \times \int_{-1}^{1}\left(1-u^{2}\right)^{k-3 / 2}\left(\frac{m+2 n+2 u \sqrt{n(n+m)}}{m}\right)^{1 / 2-k+i \kappa_{j}} d u
\end{aligned}
$$

when $K^{2-\varepsilon} \ll m \ll K^{2+\varepsilon}$. Now by Taylor's approximation we are able to separate the variables $\kappa_{j}$ and $m$ as

$$
\left(\frac{m+2 n+2 u \sqrt{n(n+m)}}{m}\right)^{i \kappa_{j}} \sim\left(\frac{m+2 n+2 u \sqrt{n(n+m)}}{m}\right)^{i K} .
$$


Finally

$$
\mathcal{P}_{s_{j}-1}^{1-k}\left(\frac{2 n+m}{|m|}\right) \sim\left(\frac{n}{n+m}\right)^{(k-1) / 2} \chi(m)
$$

with $\chi$ a smooth function satisfying the condition $\chi^{(\nu)}(m) \ll_{\nu}\left(m K^{-\varepsilon}\right)^{-\nu}$ for all $\nu \geq 0$.

While Good now proceeds with estimations by absolute values, we transform our problem to a form similar to that treated in [JM1] and [JM2]. We first insert a smooth weight function $\phi(m)$ into the $m$-sum in (3.2) and then decompose the resulting double sum into five parts over $-n+1 \leq m \ll$ $K^{2 / 3+\varepsilon}, K^{2 / 3+\varepsilon} \ll m \ll K^{4 / 3}, K^{4 / 3} \ll m \ll K^{2-\varepsilon}, K^{2-\varepsilon} \ll m \ll K^{3}$ and $m \gg K^{3}$. The first and the last case can be settled easily by estimating the $m$-sum trivially.

To deal with the second case $K^{2 / 3+\varepsilon} \ll m \ll K^{4 / 3}$, we write $\kappa_{j}=$ $K+\left(\kappa_{j}-K\right)$ and conclude by use of Taylor's approximation that

with

$$
\exp \left( \pm i \kappa_{j} \log A(m, n)\right) \sim \exp ( \pm i K \log A(m, n))
$$

$$
A(m, n)=\left(1+\frac{2 n}{m}+2 \frac{\sqrt{n(n+m)}}{m}\right)^{-1} .
$$

Thus the variables $\kappa_{j}$ and $m$ can be separated in the exponential term and by the spectral large sieve we come to the desired conclusion.

We next concentrate on proving the requested estimate for the third case $K^{4 / 3} \ll m \ll K^{2-\varepsilon}$, which turns out to be the most difficult one, and then comment briefly on the fourth case $K^{2-\varepsilon} \ll m \ll K^{3}$ at the end of this paper.

By following the same argument as in the second case it suffices to prove that for all fixed $n \in \mathbb{Z}_{+}$and $K^{4 / 3} \ll M \ll K^{2-\varepsilon}$,

$$
\begin{aligned}
\sum_{K \leq \kappa_{j} \leq K+G} \alpha_{j} \mid \sum_{m=1}^{\infty} \phi(m) t_{j}(m) & \overline{a(m+n)}(n+m)^{1 / 4-k / 2} \\
& \times\left.\exp ( \pm i K \log A(m, n))\right|^{2} \ll K^{2+1 / 3+\varepsilon}
\end{aligned}
$$

with $G=K^{1 / 3+\varepsilon}, A(m, n)$ as above, and $\phi$ a suitable smooth weight function compactly supported on the interval $[3 M / 4,2 M]$, and $\phi^{(\nu)} \ll_{\nu} M^{-\nu}$ for each $\nu \geq 0$. Note that we introduce the notation $G$ in order to make the analogy between our case and that in the papers by Jutila and Motohashi even more visible.

In what follows, we shall concentrate on the case $+i K$ and prove that

$$
\begin{aligned}
\mathcal{S}=\sum_{K \leq \kappa_{j} \leq K+G} \alpha_{j} \mid \sum_{m=1}^{\infty} \phi(m) t_{j}(m) \overline{a(m+n)}(n+m)^{1 / 4-k / 2} & \\
& \times\left.\exp \left(-i K \log A(m, n)^{-1}\right)\right|^{2}
\end{aligned}
$$


is $\ll K^{2+1 / 3+\varepsilon}$, noting that the case for $-i K$ follows easily by complex conjugation and some minor modifications.

We next simplify the exponential factor

$$
\exp \left(-i K \log A(m, n)^{-1}\right)
$$

by noticing that

$$
\log \left(1+\frac{2 n}{m}+2 \frac{\sqrt{n(n+m)}}{m}\right)=2\left(\frac{n}{m}\right)^{1 / 2}-\frac{1}{3}\left(\frac{n}{m}\right)^{3 / 2}+\cdots+\mathcal{O}\left(K^{-P}\right)
$$

with $P$ an arbitrarily large positive constant. Hence

$$
\exp \left(-i K \log A(m, n)^{-1}\right) \sim \exp \left(-2 i K \sqrt{\frac{n}{m}}\right)
$$

giving

$$
\begin{aligned}
\mathcal{S} \sim \sum_{K \leq \kappa_{j} \leq K+G} \alpha_{j} \mid \sum_{m=1}^{\infty} \phi(m) t_{j}(m) \overline{a(m+n)}(n & +m)^{1 / 4-k / 2} \\
& \times\left.\exp \left(-2 i K \sqrt{\frac{n}{m}}\right)\right|^{2} .
\end{aligned}
$$

We now have a sum similar to Jutila and Motohashi's sum (4.1) in [JM1, only there is an exponential factor instead of the factor $m^{-i t}$ with some fixed $t$ and $\overline{a(m+n)}$ in place of $d(m)$. We proceed following their argument from the beginning of their Chapter 4, p. 82, inserting factors $h\left(\kappa_{j}\right)$ with

$$
h(r)=K^{-2}\left(r^{2}+\frac{1}{4}\right)\left[\exp \left(-\left(\frac{r-K}{G}\right)^{2}\right)+\exp \left(-\left(\frac{r+K}{G}\right)^{2}\right)\right]
$$

to the $\kappa_{j}$-sum and squaring out the $m$-sum to obtain the upper bound

$$
\begin{aligned}
& \sum_{j=1}^{\infty} \alpha_{j} \sum_{m_{1}=1}^{\infty} \sum_{m_{2}=1}^{\infty} \phi\left(m_{1}\right) \phi\left(m_{2}\right) t_{j}\left(m_{1}\right) t_{j}\left(m_{2}\right) \overline{a\left(m_{1}+n\right)} a\left(m_{2}+n\right) \\
& \times\left(n+m_{1}\right)^{1 / 4-k / 2}\left(n+m_{2}\right)^{1 / 4-k / 2} \exp \left(-2 i K \sqrt{\frac{n}{m_{1}}}\right) \exp \left(2 i K \sqrt{\frac{n}{m_{2}}}\right) h\left(\kappa_{j}\right) .
\end{aligned}
$$

We then apply Lemma 2.7 and arrive at the sum

$$
\begin{aligned}
& \mathcal{S}_{1}=\sum_{m_{1}=1}^{\infty} \sum_{m_{2}=1}^{\infty} \phi\left(m_{1}\right) \phi\left(m_{2}\right) \overline{a\left(m_{1}+n\right)} a\left(m_{2}+n\right) \\
& \times\left(n+m_{1}\right)^{1 / 4-k / 2}\left(n+m_{2}\right)^{1 / 4-k / 2} \exp \left(-2 i K \sqrt{\frac{n}{m_{1}}}\right) \exp \left(2 i K \sqrt{\frac{n}{m_{2}}}\right) \\
& \times \sum_{l=1}^{\infty} \frac{\phi_{1}(l)}{l} S\left(m_{1}, m_{2} ; l\right) \hat{h}\left(\frac{4 \pi \sqrt{m_{1} m_{2}}}{l}\right),
\end{aligned}
$$

which corresponds to formula (4.2) in [JM1. Here $\phi_{1}$ is a suitable smooth 
weight function compactly supported on the interval $[3 L / 4,2 L]$ where

$$
L \ll \frac{M \log K}{K G},
$$

and $\phi_{1}^{(\nu)} \ll_{\nu} L^{-\nu}$ for each $\nu \geq 0$. The treatment of the first and the second term and the truncation of the $l$-sum in the Kloosterman-spectral sum formula can be carried out as in [JM1, pp. 74-76]. Now it is enough to prove that

$$
\mathcal{S}_{1} \ll K^{2+1 / 3+\varepsilon} .
$$

Next we find the Mellin transform $\mathcal{M}(\cdot ; s)$ for the function $e^{-i y} w(y)$ with $w$ a real-valued smooth weight function compactly supported on the interval $[T / 2,6 T]$ with $T \geq 1, w(y)=1$ on the interval $[T, 5 T]$ and $w^{(\nu)} \ll_{\nu} T^{-\nu}$ for each $\nu \geq 0$. By the saddle-point method,

$$
\mathcal{M}\left(e^{-i y} w(y) ; s\right) \sim \sqrt{2 \pi} w(t) t^{\sigma-1 / 2+i t} e^{-i t-\pi i / 4}
$$

with $s=\sigma+i t, \sigma$ in a bounded interval. Substituting this into the Mellin inversion formula for $e^{-i y} w(y)$ and moving the path of integration to $c=$ $-1 / 2$ we get

$$
\begin{aligned}
e^{-i y} w(y) & =\frac{1}{2 \pi i} \int_{(c)} \mathcal{M}\left(e^{-i y} w(y) ; s\right) y^{-s} d s \\
& \sim \frac{1}{\sqrt{2 \pi}} e^{-\pi i / 4} \int_{T / 2}^{6 T} w(t) t^{-1+i t} e^{-i t} y^{1 / 2-i t} d t .
\end{aligned}
$$

We use the notations $2 K \sqrt{n / m}=y$ and

$$
T=\frac{K \sqrt{n}}{\sqrt{M}}
$$

and transform our exponential terms in (3.3) by formula (3.4). We then use the same reasoning as in JM1] (writing out the Kloosterman sums and applying trigonometric approximations for the Bessel functions arising from the definition of $\hat{h}$ ) and finally get an analogous expression to Jutila and Motohashi's sum (4.9):

$$
\begin{aligned}
\mathcal{S}_{2}= & \sum_{m_{1}=1}^{\infty} \phi\left(m_{1}\right) \overline{a\left(m_{1}+n\right)}\left(n+m_{1}\right)^{1 / 4-k / 2} m_{1}^{-1 / 2+i t_{1}} \sum_{l=1}^{\infty} \frac{\phi_{1}(l)}{\sqrt{l}} \\
& \times \sum_{\substack{q=1 \\
(q, l)=1}}^{l} e\left(\frac{q m_{1}}{l}\right) \sum_{m_{2}=1}^{\infty} \phi\left(m_{2}\right) a\left(m_{2}+n\right)\left(n+m_{2}\right)^{1 / 4-k / 2} m_{2}^{-1 / 2-i t_{2}} \\
& \times e\left(\frac{\bar{q}\left(m_{2}+n\right)}{l}\right) e\left(\frac{-\bar{q} n}{l}\right) \exp \left(\delta_{1} i \omega\left(r, \frac{4 \pi \sqrt{m_{1} m_{2}}}{l}\right)\right),
\end{aligned}
$$


and it suffices to prove that

$$
\begin{array}{r}
\int_{T / 4}^{3 T} \int_{T / 4}^{3 T} w\left(2 t_{1}\right) w\left(2 t_{2}\right)\left(2 t_{1}\right)^{-1+2 i t_{1}}\left(2 t_{2}\right)^{-1-2 i t_{2}} e^{-2 i t_{1}+2 i t_{2}}(2 K)^{-2 i t_{1}+2 i t_{2}} \\
\times n^{-i t_{1}+i t_{2}} \mathcal{S}_{2} d t_{1} d t_{2} \ll K^{\varepsilon}
\end{array}
$$

Here $\delta_{1}= \pm 1, q \bar{q} \equiv 1(\bmod l),|r-K| \leq G \log K$ and

$$
\omega(r, x)=x\left(1-2\left(\frac{r}{x}\right)^{2}\right) .
$$

Note that we have $t_{1}$ and $t_{2}$ instead of one $t$ in JM1. Notice also that we replaced $t$ by $2 t$ in (3.4) in order to make the analogy even more visible.

Next we apply the sum formula of Voronor, which can be found, for example, in Jutila's monograph [J1, Theorem 1.7], to the inner-most sum over $m_{2}$. Note that we may write the function

$$
J_{k-1}\left(\frac{4 \pi \sqrt{m_{2}(y+n)}}{l}\right),
$$

which appears in this formula, asymptotically in terms of functions

$$
\frac{1}{\sqrt{2} \pi} l^{1 / 2}\left(m_{2}(y+n)\right)^{-1 / 4} \exp \left(\delta_{2} i \frac{4 \pi \sqrt{m_{2}(y+n)}}{l}\right) \exp \left(-\delta_{2} i \pi(k-1) / 2\right),
$$

$\delta_{2}= \pm 1$ (see Lebedev $[\mathrm{L}$, eq. (5.11.6)]). Here $y$ is the variable of integration arising from the sum formula. Furthermore we proceed as in the paper by Jutila and Motohashi, arriving at the sum

$$
\begin{aligned}
\mathcal{S}_{3}=\sum_{f \asymp f_{0}} \sum_{l=1}^{\infty} \frac{\phi_{1}(l) S(f, n ; l)}{l} \sum_{m=1}^{\infty} \phi(m) \overline{a(m+n)} a(m+f) \\
\times(n+m)^{1 / 4-k / 2} m^{-1 / 2+i t_{1}}(m+f)^{1 / 4-k / 2} \int_{0}^{\infty} \phi(y)(y+n)^{-1 / 2} y^{-1 / 2-i t_{2}} \\
\quad \times \exp \left(\delta_{1} i \omega\left(r, \frac{4 \pi \sqrt{m y}}{l}\right)-\delta_{1} i \frac{4 \pi \sqrt{(m+f)(y+n)}}{l}\right) d y
\end{aligned}
$$

with

$$
f_{0} \asymp L^{2} T^{2} .
$$

This corresponds to the sum $\mathcal{S}_{3}^{-}$in [JM1]. Now it suffices to prove that

$$
\begin{aligned}
\int_{T / 4}^{3 T} \int_{T / 4}^{3 T} w\left(2 t_{1}\right) w\left(2 t_{2}\right)\left(2 t_{1}\right)^{-1+2 i t_{1}}\left(2 t_{2}\right)^{-1-2 i t_{2}} e^{-2 i t_{1}+2 i t_{2}}(2 K)^{-2 i t_{1}+2 i t_{2}} & \\
& \times n^{-i t_{1}+i t_{2}} \mathcal{S}_{3} d t_{1} d t_{2} \ll K^{\varepsilon} .
\end{aligned}
$$


Notice that since now $t_{2} \asymp T$, we get the order of magnitude of $f$ by a saddlepoint analysis of the $y$-integral, and we find that the sum corresponding to $\mathcal{S}_{3}^{+}$in [JM1] is negligibly small. Notice also that, in our case, the Kloosterman sum $S(f, n ; l)$ appears instead of the Ramanujan sum $c_{l}(f)=S(f, 0 ; l)$.

Now in order to make the analogy to [JM1] more visible we substitute $f=\tilde{f}+n$ and $m=\tilde{m}-n$ into (3.5) to obtain

$$
\begin{aligned}
\mathcal{S}_{3} \sim & \sum_{\tilde{f} \asymp f_{0}} \sum_{l=1}^{\infty} \frac{\phi_{1}(l) S(\tilde{f}+n, n ; l)}{l} \sum_{\tilde{m}=1}^{\infty} \tilde{\phi}(\tilde{m}) \overline{a(\tilde{m})} a(\tilde{m}+\tilde{f}) \\
& \times \tilde{m}^{-1 / 4-k / 2+i t_{1}}(\tilde{m}+\tilde{f})^{1 / 4-k / 2} \int_{0}^{\infty} \phi(y) y^{-1-i t_{2}} \\
& \times \exp \left(\delta_{1} i\left(\frac{4 \pi \sqrt{\tilde{m} y}}{l}-\frac{r^{2} l}{2 \pi \sqrt{\tilde{m} y}}-\frac{4 \pi \sqrt{(\tilde{m}+\tilde{f}) y}}{l}\right)\right) d y
\end{aligned}
$$

with $\tilde{\phi}(\tilde{m})=\phi(\tilde{m}-n)$ a smooth weight function similar to $\phi$. For the sake of simplicity, we replace $\tilde{m}$ and $\tilde{f}$ by $m$ and $f$ again and continue to follow the steps in [JM1] arriving at

$$
\begin{aligned}
\mathcal{S}_{4}=(4 \pi)^{2 i t_{2}} \sum_{f=1}^{\infty} \frac{\phi_{2}(f)}{f^{k-i\left(t_{1}+t_{2}\right)}} \sum_{l=1}^{\infty} \frac{\phi_{1}(l) S(f+n, n ; l)}{l^{1+2 i t_{2}}} \\
\times \sum_{m=1}^{\infty} \overline{a(m)} a(m+f) X\left(\frac{m}{f}\right) .
\end{aligned}
$$

Here $\phi_{2}$ is a characteristic function of the interval $[F, 2 F]$ with $F \asymp L^{2} T^{2}$. Moreover,

$$
X(u)=u^{-k+i\left(t_{1}-t_{2}\right)} \int_{0}^{\infty} v^{-1-2 i t_{2}} \xi(f, l, u, v) \exp \left(-\delta_{1} i Y\right) d v
$$

with

$$
\xi(f, l, u, v)=\tilde{\phi}(f u) \phi\left(\frac{(l v)^{2} u}{16 \pi^{2} f}\right) \quad \text { and } \quad Y=\frac{1}{2} v+\frac{2 r^{2}}{u v}
$$

Now

$$
u \asymp M / F \text { and } v \asymp F / L .
$$

Again it is enough to prove that

$$
\begin{aligned}
& \int_{T / 4}^{3 T} \int_{T / 4}^{3 T} w\left(2 t_{1}\right) w\left(2 t_{2}\right)\left(2 t_{1}\right)^{-1+2 i t_{1}}\left(2 t_{2}\right)^{-1-2 i t_{2}} e^{-2 i t_{1}+2 i t_{2}}(2 K)^{-2 i t_{1}+2 i t_{2}} \\
& \times n^{-i t_{1}+i t_{2}} \mathcal{S}_{4} d t_{1} d t_{2} \ll K^{\varepsilon}
\end{aligned}
$$


Further we use Lemma 2.6 and spectrally decompose the shifted convolution in (3.6) (cf. Lemma 5 in [JM1]) and follow the estimation of the term $S_{d}$ in the case of the lower range in [JM1, pp. 92-93]. We concentrate in the further text on the first term on the right hand side of (2.6) and the factor $\Phi_{k}\left(\kappa_{j} ; W\right)$ without the term $\left(\kappa_{j} \mapsto-\kappa_{j}\right)$, the treatment of the other cases being similar or easier. We arrive at

$$
\begin{aligned}
\mathcal{S}_{5}=(4 \pi)^{2 i t_{2}} & \sum_{f=1}^{\infty} \frac{\phi_{2}(f)}{f^{1 / 2-i\left(t_{1}+t_{2}\right)}} \sum_{l=1}^{\infty} \frac{\phi_{1}(l) S(f+n, n ; l)}{l^{1+2 i t_{2}}} \sum_{\kappa_{j} \asymp L T^{2}} \overline{\rho_{j}} t_{j}(f) c_{j} \\
& \times \frac{1}{\sinh \left(\pi \kappa_{j}\right)} \frac{\Gamma\left(1 / 2+i \kappa_{j}\right)}{\Gamma\left(k-1 / 2-i \kappa_{j}\right) \Gamma\left(1+2 i \kappa_{j}\right)} \Xi_{1}\left(f, l, \kappa_{j}, \delta_{1}\right)
\end{aligned}
$$

with

$$
\Xi_{1}\left(f, l, \kappa_{j}, \delta_{1}\right)=\int_{0}^{\infty} \int_{0}^{\infty} \xi(f, l, u, v) \exp \left(-\delta_{1} i Y\right) u^{-3 / 2-i\left(\kappa_{j}-t_{1}+t_{2}\right)} v^{-1-2 i t_{2}} d u d v
$$

corresponding to expressions (5.21) (without the absolute values) and (5.22) in JM1. The condition for $\kappa_{j}$ follows by a saddle-point argument. Finally it suffices to prove that

$$
\begin{aligned}
\int_{T / 4}^{3 T} \int_{T / 4}^{3 T} w\left(2 t_{1}\right) w\left(2 t_{2}\right)\left(2 t_{1}\right)^{-1+2 i t_{1}}\left(2 t_{2}\right)^{-1-2 i t_{2}} e^{-2 i t_{1}+2 i t_{2}}(2 K)^{-2 i t_{1}+2 i t_{2}} & \\
& \times n^{-i t_{1}+i t_{2}} \mathcal{S}_{5} d t_{1} d t_{2} \ll K^{\varepsilon}
\end{aligned}
$$

Again we use the Mellin inversion to obtain

$$
\begin{aligned}
& \Xi_{1}\left(f, l, \kappa_{j}, \delta_{1}\right)=\frac{\delta_{1} i}{\pi^{2} 4^{1+i t_{2}} r^{1+2 i\left(\kappa_{j}-t_{1}+t_{2}\right)}} \int_{(0)} \int_{(0)}(\tilde{\phi})^{*}\left(s_{1}\right) \phi^{*}\left(s_{2}\right) f^{-s_{1}+s_{2}} \\
& \times\left(\frac{2 \pi}{l}\right)^{2 s_{2}} r^{-2 s_{1}-2 s_{2}} \exp \left(-\delta_{1} i \pi\left(s_{1}+i \kappa_{j}-i t_{1}\right)\right) \\
& \times \Gamma\left(1 / 2+s_{1}-s_{2}+i\left(\kappa_{j}-t_{1}-t_{2}\right)\right) \Gamma\left(1 / 2+s_{1}+s_{2}+i\left(\kappa_{j}-t_{1}+t_{2}\right)\right) d s_{1} d s_{2}
\end{aligned}
$$

with $(\tilde{\phi})^{*}$ and $\phi^{*}$ the Mellin transforms of the respective functions. This corresponds to formula (5.24) in JM1.

Next we represent the weight function $\phi_{1}(l)$ by its Mellin inversion

$$
\frac{1}{2 \pi i} \int_{(1 / 2+\varepsilon)} \phi_{1}^{*}\left(s_{3}\right) l^{-s_{3}} d s_{3}
$$

and transform the $l$-sum spectrally by Lemma 2.2. We shall concentrate on estimating the first term on the right hand side of (2.4) and comment briefly on the remaining terms later. To avoid confusion with the summation over the variable $\kappa_{j}$ in (3.7), we denote the new variable of summation by $\kappa_{j^{\prime}}$. 
By Stirling's formula, we notice that we may assume that $\kappa_{j^{\prime}} \ll T K^{\varepsilon}$. Further we may truncate the integrals over $s_{i}$ to the intervals $\operatorname{Im} s_{i}=\gamma_{i}$ $\ll K^{\varepsilon}$, since the functions $(\tilde{\phi})^{*}, \phi^{*}$ and $\phi_{1}^{*}$ are of rapid decay in $\gamma_{i}, i=1,2,3$, respectively. Lastly, we move the $s_{3}$-integration to the line $(\varepsilon)$. Finally, after applying Cauchy's inequality to the $\kappa_{j}$-sum, and Jutila's first estimate in (1.1) for a long interval, it remains to show that

$$
\begin{aligned}
\sup _{\gamma_{1}, \gamma_{2}, \gamma_{3} \ll K^{\varepsilon}}\left(L T^{2}\right)^{1 / 2} \\
\times\left(\sum_{\kappa_{j} \asymp L T^{2}} \alpha_{j} \mid \sum_{f} \phi_{2}(f) f^{-1 / 2-i \gamma_{1}+i \gamma_{2}}(f+n)^{-i \gamma_{2}-\varepsilon / 2-i \gamma_{3} / 2} t_{j}(f)\right. \\
\quad \times \sum_{\kappa_{j^{\prime}}} \frac{\overline{\rho_{j^{\prime}}(f+n)} \rho_{j^{\prime}}(n)}{\cosh \left(\pi \kappa_{j^{\prime}}\right)} \int_{T / 4 T / 4}^{3 T} \int_{T / 4}^{3 T} w\left(2 t_{1}\right) w\left(2 t_{2}\right)\left(2 t_{1}\right)^{-1+2 i t_{1}}\left(2 t_{2}\right)^{-1-2 i t_{2}} \\
\times e^{-2 i t_{1}+2 i t_{2}}\left(\frac{2 K \sqrt{f}}{r \sqrt{f+n}}\right)^{2 i t_{2}}\left(\frac{r \sqrt{f}}{2 K \sqrt{n}}\right)^{2 i t_{1}} \\
\times \Gamma\left(1 / 2+i\left(\gamma_{1}-\gamma_{2}+\kappa_{j}-t_{1}-t_{2}\right)\right) \Gamma\left(1 / 2+i\left(\gamma_{1}+\gamma_{2}+\kappa_{j}-t_{1}+t_{2}\right)\right) \\
\times \Gamma\left(\varepsilon / 2+i\left(t_{2}+\gamma_{2}+\gamma_{3} / 2+\kappa_{j^{\prime}}\right)\right) \Gamma\left(\varepsilon / 2+i\left(t_{2}+\gamma_{2}+\gamma_{3} / 2-\kappa_{j^{\prime}}\right)\right) \\
\left.\times\left.\exp \left(\delta_{1} \pi\left(\gamma_{1}+\kappa_{j}-t_{1}\right)\right) \exp \left(\delta_{3} \pi\left(t_{2}+\gamma_{2}+\gamma_{3} / 2\right)\right) d t_{1} d t_{2}\right|^{2}\right)^{1 / 2}
\end{aligned}
$$

is $\ll K^{1+\varepsilon}$. The constant $\delta_{3}$ is \pm 1 . Now we write $\Gamma(s)=\exp (\log \Gamma(s))$, use Taylor's approximation and derive an estimate of the $\nu$ th derivative of $\log \Gamma(s)$ from the asymptotic expansion $(2.2)$. Differentiation on both sides $\nu$ times can be justified by the use of Cauchy's integral formula. We therefore conclude that

$$
\begin{aligned}
\Gamma\left(1 / 2+i\left(\gamma_{1}-\gamma_{2}+\kappa_{j}-t_{1}-t_{2}\right)\right) \Gamma\left(1 / 2+i\left(\gamma_{1}\right.\right. & \left.\left.+\gamma_{2}+\kappa_{j}-t_{1}+t_{2}\right)\right) \\
& \sim \Gamma^{2}\left(1 / 2+i\left(\gamma_{1}+\kappa_{j}-t_{1}\right)\right),
\end{aligned}
$$

so we are able to separate the $t_{1}$ - and $t_{2}$-integrals from each other. Further we divide the $\kappa_{j}$-sum into subsums of interval length $L T$ and fix a variable $\kappa$ in each of these intervals, so that we always have

$$
\frac{\kappa_{j}-\kappa}{\kappa} \ll \frac{1}{T} .
$$

Now we multiply the $t_{1}$-integral by the factor

$$
\exp \left(-2 i \kappa_{j} \log \kappa_{j}+2 i \kappa_{j}\right)
$$

and conclude by (2.1) and repeated use of Taylor's approximation that

$$
\begin{aligned}
\Gamma^{2}\left(1 / 2+i\left(\gamma_{1}+\kappa_{j}-t_{1}\right)\right) e^{-2 i \kappa_{j} \log \kappa_{j}+2 i \kappa_{j}} & \sim 2 \pi e^{2 i\left(\gamma_{1}-t_{1}\right) \log \kappa_{j}} e^{-\pi\left(\gamma_{1}+\kappa_{j}-t_{1}\right)} \\
& \sim 2 \pi e^{2 i\left(\gamma_{1}-t_{1}\right) \log \kappa} e^{-\pi\left(\gamma_{1}+\kappa_{j}-t_{1}\right)} .
\end{aligned}
$$


Therefore we may ignore the dependence of the $t_{1}$-integral on $\kappa_{j}$ and use the spectral large sieve for the $\kappa_{j}$-sum. We then apply either the second derivative test (see e.g. Ivić [Iv1, Lemma 2.2]) or trivial estimation to the $t_{1^{-}}$and $t_{2}$-integrals and finally end our proof of this case.

The second term on the right hand side of (2.4) is treated similarly, this case being even easier, and the last term obviously equals zero in our case. In the third term we denote the variable of summation by $k^{\prime}$ to distinguish it from the weight $k$. We first treat the part of the $k^{\prime}$-sum where $k^{\prime} \gg$ $T K^{\varepsilon}$. We move the $s_{3}$-integral into the $k^{\prime}$-sum shifting the integration to the line $-P$ with $P$ a suitably large constant, and then apply the functional equation $\Gamma(z+1)=z \Gamma(z)$ to the $\Gamma$-function in the denominator. By trivial estimations we see that this part of the $k^{\prime}$-sum is negligibly small. For the part where $k^{\prime} \ll T K^{\varepsilon}$ we move the $s_{3}$-integral to the imaginary axis and end up estimating

$$
\begin{aligned}
\sup _{\gamma_{1}, \gamma_{2}, \gamma_{3} \ll K^{\varepsilon}}\left(L T^{2}\right)^{1 / 2}\left(\sum_{\kappa_{j} \asymp L T^{2}} \alpha_{j} \mid \sum_{f} \phi_{2}(f) f^{-1 / 2-i \gamma_{1}+i \gamma_{2}}(f+n)^{-i \gamma_{2}-i \gamma_{3} / 2} t_{j}(f)\right. \\
\quad \times \sum_{k^{\prime} \ll T K^{\varepsilon}}\left(2 k^{\prime}-1\right) q_{f+n, n}\left(k^{\prime}\right) \int_{T / 4 T / 4}^{3 T} w\left(2 t_{1}\right) w\left(2 t_{2}\right)\left(2 t_{1}\right)^{-1+2 i t_{1}}\left(2 t_{2}\right)^{-1-2 i t_{2}} \\
\quad \times e^{-2 i t_{1}+2 i t_{2}}\left(\frac{2 K \sqrt{f}}{r \sqrt{f+n}}\right)^{2 i t_{2}}\left(\frac{r \sqrt{f}}{2 K \sqrt{n}}\right)^{2 i t_{1}} \\
\times \Gamma\left(1 / 2+i\left(\gamma_{1}-\gamma_{2}+\kappa_{j}-t_{1}-t_{2}\right)\right) \Gamma\left(1 / 2+i\left(\gamma_{1}+\gamma_{2}+\kappa_{j}-t_{1}+t_{2}\right)\right) \\
\left.\times\left.\frac{\Gamma\left(k^{\prime}-1 / 2+i\left(t_{2}+\gamma_{2}+\gamma_{3} / 2\right)\right)}{\Gamma\left(k^{\prime}+1 / 2-i\left(t_{2}+\gamma_{2}+\gamma_{3} / 2\right)\right)} \exp \left(\delta_{1} \pi\left(\gamma_{1}+\kappa_{j}-t_{1}\right)\right) d t_{1} d t_{2}\right|^{2}\right)^{1 / 2}
\end{aligned}
$$

The $t_{i}$-integrals can be treated as before. For the $t_{2}$-integral, we write $\Gamma(s)=$ $\exp (\log \Gamma(s))$ and notice that for $s=\sigma+i t$,

$$
\frac{d^{2}}{d t^{2}} \operatorname{Im} \log \Gamma(s)=\operatorname{Im} \frac{d^{2}}{d t^{2}} \log \Gamma(s)=\frac{-t}{|s|^{2}}+\mathcal{O}\left(|s|^{-2}\right)
$$

by the asymptotic expansion for $\frac{d^{2}}{d s^{2}} \log \Gamma(s)$ obtained from 2.2 .

Finally, the case $K^{2-\varepsilon} \ll m \ll K^{3}$ can essentially be treated in the same way as the case $K^{4 / 3} \ll m \ll K^{2-\varepsilon}$ above, without the exponential term and therefore without the $t_{i}$-integrals. Now it suffices to prove that

$$
\begin{aligned}
\mathcal{S}=\sum_{K \leq \kappa_{j} \leq K+G} \alpha_{j} \mid \sum_{m=1}^{\infty} \tilde{\phi}(m) t_{j}(m) \overline{a(m+n)}(n+m)^{1 / 4-k / 2} & \\
& \times\left. m^{-1 / 4}\left(\frac{m}{M}\right)^{-1 / 4}\left(\frac{n+m}{M}\right)^{3 / 4-k / 2}\right|^{2} M^{1-k}
\end{aligned}
$$


is $\ll K^{-2 k+3+1 / 3+\varepsilon}$. Here $\tilde{\phi}$ is a smooth weight function compactly supported on the interval $[3 M / 4,2 M]$, and $\phi^{(\nu)} \ll_{\nu}\left(M K^{-\varepsilon}\right)^{-\nu}$ for each $\nu \geq 0$. Further we may embed the term

$$
\left(\frac{m}{M}\right)^{-1 / 4}\left(\frac{n+m}{M}\right)^{3 / 4-k / 2} \ll 1
$$

into $\tilde{\phi}$ having a similar situation to the case $K^{4 / 3} \ll M \ll K^{2-\varepsilon}$ with the exception that now $\tilde{\phi}^{(\nu)} \ll_{\nu}\left(M K^{-\varepsilon}\right)^{-\nu}$. However, this does not cause any new difficulties. See also [JM1, p. 83], where Jutila and Motohashi have $\phi^{(\nu)} \ll_{\nu}\left(M \log ^{-4} K\right)^{-\nu}$.

We proceed as above, until we reach $\mathcal{S}_{3}$ and estimate trivially the case $L \ll K^{\varepsilon}+M K^{-2+\varepsilon}$ using the bound (2.3). This time we insert a smooth $\phi_{2}$ instead of a characteristic function. Finally, as we reach the point where we represented the weight function $\phi_{1}$ by its Mellin inversion, to estimate the remaining $l$-sum we use this time Lemma 2.3 and conclude with the desired result.

Acknowledgements. The author would like to thank Professor Matti Jutila for the idea for this subject as well as for his helpful comments and suggestions. This research was supported by Finnish Cultural Foundation.

\section{References}

[BR1] J. Bernstein and A. Reznikov, Periods, subconvexity of L-functions and representation theory, J. Differential Geom. 70 (2005), 129-141.

[BR2] - - - Subconvexity bounds for triple L-functions and representation theory, arXiv:math.NT/0608555.

[D] P. Deligne, La conjecture de Weil. I, Inst. Hautes Études Sci. Publ. Math. 43 (1974), 273-307.

[EMOT] A. Erdélyi, W. Magnus, F. Oberhettinger and F. G. Tricomi, Higher Transcendental Functions, Vol. 1, McGraw-Hill, New York, 1953.

[Es] T. Estermann, On Kloosterman's sum, Mathematika 8 (1961), 83-86.

[G1] A. Good, Beiträge zur Theorie der Dirichletreihen, die Spitzenformen zugeordnet sind, J. Number Theory 13 (1981), 18-65.

[G2] - Cusp forms and eigenfunctions of the Laplacian, Math. Ann. 255 (1981), 523-548.

[He] D. A. Hejhal, The Selberg Trace Formula for PSL(2, R), Vol. 2, Lecture Notes in Math. 1001, Springer, Berlin, 1983.

[HL] J. Hoffstein and P. Lockhart, Coefficients of Maass forms and the Siegel zero, Ann. of Math. 140 (1994), 161-181.

[Iv1] A. Ivić, The Riemann Zeta-Function. The Theory of the Riemann Zeta-Function with Applications, Wiley, New York, 1985.

[Iv2] - On sums of Hecke series in short intervals, J. Théor. Nombres Bordeaux 13 (2001), 453-468. 
[Iw1] H. Iwaniec, Mean values for Fourier coefficients of cusp forms and sums of Kloosterman sums, in: Journées Arithmétiques, 1980 (Exeter, 1980), J. V. Armitage (ed.), London Math. Soc. Lecture Note Ser. 56, Cambridge Univ. Press, Cambridge, 1982, 306-321.

[Iw2] - The spectral growth of automorphic L-functions, J. Reine Angew. Math. 428 (1992), 139-159.

[J1] M. Jutila, Lectures on a Method in the Theory of Exponential Sums, Tata Inst. Fund. Res. Lectures Math. Phys. 80, Springer, Berlin, 1987.

[J2] - The additive divisor problem and its analogs for Fourier coefficients of cusp forms. I, Math. Z. 223 (1996), 435-461.

[J3] - The additive divisor problem and its analogs for Fourier coefficients of cusp forms. II, ibid. 225 (1997), 625-637.

[J4] - The fourth moment of central values of Hecke series, in: Number Theory. Proc. Turku Sympos. on Number Theory in Memory of Kustaa Inkeri (Turku, 1999), M. Jutila and T. Metsänkylä (eds.), de Gruyter, Berlin, 2001, 167-177.

[J5] -, On spectral large sieve inequalities, Funct. Approx. Comment. Math. 28 (2000), 7-18.

[JM1] M. Jutila and Y. Motohashi, Uniform bound for Hecke L-functions, Acta Math. 195 (2005), 61-115.

[JM2] -, 一, Uniform bounds for Rankin-Selberg L-functions, in: Multiple Dirichlet Series, Automorphic Forms, and Analytic Number Theory (Bretton Woods, NH, 2005), S. Friedberg et al. (eds.), Proc. Sympos. Pure Math. 75, Amer. Math. Soc., Providence, RI, 2006, 243-256.

[K] N. V. Kuznetsov, Petersson's hypothesis for parabolic forms of weight zero and Linnik's hypothesis. Sums of Kloosterman sums, Mat. Sb. 111 (153) (1980), 334-383 (in Russian); English transl.: Math. USSR-Sb. 39 (1981), 299-342.

[L] N. N. Lebedev, Special Functions and Their Applications, Dover, New York, 1972.

[Me] T. Meurman, On exponential sums involving the Fourier coefficients of Maass wave forms, J. Reine Angew. Math. 384 (1988), 192-207.

[Mo1] Y. Motohashi, The binary additive divisor problem, Ann. Sci. École Norm. Sup. 27 (1994), 529-572.

[Mo2] - , The mean square of Hecke L-series attached to holomorphic cusp-forms, Sūrikaisekikenkyūsho Kōkyūroku 886 (1994), 214-227.

[Mo3] - , Spectral Theory of the Riemann Zeta-Function, Cambridge Univ. Press, Cambridge, 1997.

[O] F. W. J. Olver, Asymptotics and Special Functions, Academic Press, New York, 1974.

[R] R. A. Rankin, Contributions to the theory of Ramanujan's function $\tau(n)$ and similar arithmetical functions II. The order of Fourier coefficients of integral modular forms, Math. Proc. Cambridge Philos. Soc. 35 (1939), 357-372.

[San] A. Sankaranarayanan, Fundamental properties of symmetric square L-functions. I, Illinois J. Math. 46 (2002), 23-43.

[Sar] P. Sarnak, Integrals of products of eigenfunctions, Int. Math. Res. Not. 6 (1994), $251-260$.

[Se1] A. Selberg, Bemerkungen über eine Dirichletsche Reihe, die mit der Theorie der Modulformen nahe verbunden ist, Arch. Math. Naturvid. 43 (1940), 47-50.

[Se2] - On the estimation of Fourier coefficients of modular forms, in: Proc. Sympos. Pure Math. 8, Amer. Math. Soc., Providence, RI, 1965, 1-15. 
[Su] E. Suvitie, On inner products involving holomorphic cusp forms and Maass forms, PhD thesis, TUCS Dissertations Ser. 123, 2009; https://oa.doria.fi/ handle/10024/47596

Eeva Suvitie

Department of Mathematics

University of Turku

20014 Turun yliopisto, Finland

E-mail: eevsuv@utu.fi 